\title{
Hip-hop classes effect on lateral dominance indicator change among the non-sports university students
}

\author{
Yuliya Serikova ${ }^{1 *}$, and Veronika Aleksandrova ${ }^{2}$ \\ ${ }^{1}$ Senior Lecture at the Department of «Physical education», MIREA - Russian Technological \\ University, RUSSIAN FEDERATION \\ ${ }^{2}$ The candidate of pedagogical Sciences, associate Professor, Department of Adaptology and Sports \\ Training, Moscow city university, RUSSIAN FEDERATION
}

\begin{abstract}
The goal of this work to evaluate the efficiency of using a hiphop class method during the educational process of non-sports university female students based on lateral dominance indicator manifestation. One of the most important evaluation parameters of brain hemispheres activity is lateral organization profile presence or absence. Brain lateralization is manifested as a consequence if the brain hemispheres functional asymmetry occurs. Unequal distribution of brain strain on the brain hemispheres' activity is one of the obvious reasons of both motor and sensory asymmetry occurrence among those, who go in for physical training and sports. This is connected with unilateral character of the training process in sports and in physical training educational process. In the course of pedagogical testing of non-sports university female students, functional motor asymmetry with an individual right-hand type of the lateral profile has been revealed. This fact emphasizes insufficiency of impact on one of the coordination skills indicators in the process of physical training classes with students, which aggravates the processes of brain hemispheres functioning, which, in its turn, leads to inferior development of psychomotor qualities, which manifestation level is directly connected with coordination skills. As a result of the pedagogical experiment, based on using hip-hop as a means of coordination skills refinement, experimental group showed reliable improvement of lateral dominance indicator towards its decrease.
\end{abstract}

\section{Introduction}

Coordination skills, being one of the main movement characteristics, present an ability to purposefully coordinate movements, a skill of rearranging previously mastered movements, changing their parameters or switching according to changing conditions $[7,8]$. Coordination skills present an assembly of morphofunctional human body qualities, which are based on an extensive factors' complex. Coordination skills manifestation is limited by central nervous system activity specifics, i.e.: mobility and stability of neural excitation

* Corresponding author: miss17.91@ mail.ru 
and inhibition, cerebral cortex formation peculiarities, several sensor system and neuromuscular systems development level, their individual manifestation and functioning, psychomotor skills performance and psychic processes speed (memory, attention, sensing, imagination, perception, thinking), higher nervous activity type (temperament), character, and also psychic states self-adjustment $[10,11]$. Along with that, coordination skills are largely determined by heredity. There are three main groups of factors, which determine coordination skills manifestation: commensuration and regulation of movement space, time and dynamics characteristic, maintaining posture and executing a movement without excessive muscle tension, maintaining fixed posture in static positions, maintaining balance while moving $[7,8]$. High level of coordination skills manifestation and, consequently, successful execution of the set movement goal, is determined by accomplishment of central nervous system functioning mechanisms.

One of parameters, which determines high results in physical and sports activities, is a brain lateral organization profile. The stated characteristic reflects one of the most important parts of movement coordination skills. Functional brain hemispheres asymmetry is the main determining reason for developing of a certain lateral phenotype (brain lateralization as a process presents a connection between different functions and processes from one and the other brain sides). [1,5,6]. Brain hemisphere asymmetry refers to a complex brain quality, which reflects differences in neuro psychic functions distribution between the right and the left hemisphere. [5]. Lateral organization profile is developed as a combination of hemispheres' functional asymmetry, motor and sensory asymmetry. Sensory asymmetry is sense organs functioning asymmetry, motor asymmetry is asymmetry in arms, legs, facial muscles functioning. [4]. The leading hand manifests brain hemisphere dominance, in view of this, we speak of a leading hemisphere $[4,9]$.

It has been proved by the specialists, that brain interhemispheric organization influences physical skills development, depending on the purposeful sports activities specifics. Research papers confirm that at different studying stages students, who practice one focused type of sports, show motor asymmetry manifestation, expressed by leading right or left hand (e.g. in wrestling), legs (running or football), they also show manifestations of sensory asymmetry, expressed by the leading eye in shooting or tennis. [5, 11]. A certain correlation between lateral organization profile types and sports activities specifics is stated. Manifestations of motor and sensory asymmetry, specific for a particular sport type, promote achievement of high sports results [5]. It should be noted that with sport mastery increases and a more concentrated training, sensory and motor asymmetries make progress, having impact on manifestation and distribution of psychic functions [12, 14]. A marked manifestation of asymmetry is expressed not only at the level of brain activity and consequent psychic functions, which extend to sensory and motor activities, but also has a direct impact on emotional-volitional sphere of a person. Its type and an expression degree affect individual behaviour patterns. [12-15].

Still, based on physical education goals at the university, to achieve education process balance, it is necessary to exclude one-sided exposure to interhemispheric brain activity during physical and sports activities, as its leads to motor and sensory asymmetry progression. It results in decrease of motor coordination skills, which have a direct connection to psychomotor abilities, which also determine the quality of future specialists' professional activity.

To evaluate the efficiency of using hip-hop during the educational process of female students of a non-sports university based on the level of lateral dominance manifestation rate. 


\section{Research methods}

Method of analysis and generalization of scientific-methodological literature; pedagogical testing; mathematic statistics methods, pedagogical experiment.

Research procedure. The research took place in the Russian Technological University, research subjects consisted of female students of first year of studying, aged 18-20 years old, referred to the main health group, who studied the following disciplines: "Analytical Chemistry», «Applied Computer Science in Chemistry», «Biotechnology». Forty people were tested.

\section{Research results and discussion}

Pedagogical testing. To evaluate the initial level of lateral dominance and to define the lateral profile of motor asymmetry, and also these indicators' change level as the result of hip-hop used during the physical education classes with female students, we used a testing task «Juggling» and the testing task «A coordination routine».

A testing task «Juggling». Tested people were offered to throw a tennis ball to the floor with their right hand during 30 seconds, while shifting the ball from the right hand to the left hand on the rebound, and to catch the ball with their left hand after the rebound. The same should be done with the leading left hand for 30 seconds. The number of the correct throws without turnover and the difference between right-hand and left-hand variants were taken into consideration.

A testing task «Coordination routine». The testing combination consisted of classic aerobics basic steps. The testing was conducted by a stream-line (continuous) method. The chosen music corresponded to a medium to high intensity. Every five minutes the music tempo was accelerated from 120 beats per minute to 135 beats per minute. The experts registered faultless, self-dependent fulfillment of each research stage by a tested person over time. The following indicators of the task fulfillment were evaluated: hands movements (right and left) ranged from 1 to 5 points, legs movements (from the right, from the left) from 1 to 5 points, added complexity of the routine (tempo acceleration, adding rotations and right-side and left-side turning) from 1 to 5 points, total sum of all points based on all the parameters [2,3].

The following results were obtained in the course of the pedagogical testing aimed at defining the initial lateral dominance indicator in the control and experimental groups:

Based on the testing task "Juggling" in the control group the mean value of the number of correct right-hand throws was $33 \pm 0.5$ times; the number of correct left-hand throws comprised $29.3 \pm 0.5$ times; the mean value of right hand and left hand difference among the tested participants from the control group was $3.8 \pm 0.6$ (number module). In the experimental group the mean value of the number of correct right-hand throws was $32.1 \pm 0.6$ times; the number of correct left-hand throws comprised $28.4 \pm 0.5$ times; the mean value of right hand and left hand difference among the tested participants from the experimental group was $4.1 \pm 0.4$ times (number module).

Based on the test results, it was defined that the predominant lateral organization type in control and experimental groups was the right-sided type $-85 \%$, left-sided type $-7 \%$, and also $8 \%$ of the tested students were revealed as having no hand motor asymmetry.

Based on the testing task «Coordination routine» the following was revealed: the mean value for a "leg" indicator in the control group comprised $2.6 \pm 0.1$ points out of maximum 5 points; in the experimental group it comprised $2.6 \pm 0.1$ points; difficulties of mastering basic steps by a stream line method and left-direction leg movements were observed: when moving and turning to the left. 
The mean value for "hand" indicator in the control group was $2.6 \pm 0.1$ points out of maximum 5 points; in the experimental group - $2.6 \pm 0.1$ points; based on this indicator, mistakes when changing the task to the opposite side direction were revealed among the fixed right-side type.

Based on the "legs + hands" indicator in the control group the mean value comprised $2.3 \pm 0.2$ points out of maximum 5 points, in the experimental group $-2.1 \pm 0.2$ points. Disruption in coordinating hands and legs alignment was revealed, there emerged difficulties during moving and turning; doing a routine to the left with coordinating hands to the left or to the opposite side was the most difficult for the participants.

As a result, the mean value of the total point in the control group comprised $7.4 \pm 0.3$ points out of maximum 15 points, in the experimental group $-7.3 \pm 0.2$ points. The level of ability to acquire and learn new movement activities, reorganizing them while movement parameters change with altering the leading side (beginning of the routine from the other leg, in the opposite direction) with tempo increase can be estimated as "below average". The right-side motor asymmetry type was revealed.

Testing, conducted before the method was used in the education process, didn't reveal significant difference of the studied indicators between the control and experimental groups $(\mathrm{p}>0,05)$.

As the result of initial level test of lateral dominance manifestation in control and experimental group, functional motor asymmetry with the predominant right-side type was revealed. The obtained data point to the lack of variety among the methods and tools used in the process of physical education, those methods and tools could have promoted an even impact on the brain interhemispheric activity. Due to the fact that similar functioning of both brain hemispheres is considered to be physiologically normal state, in our opinion, it is necessary to introduce such forms and types of physical fitness, which will enable achieving harmonious brain interhemispheric activity, into the physical education process.

To achieve improvement of coordination skills, in particular, improving lateral dominance indicator and arms and legs coordination, a hip-hop based method was introduced to the physical education process of the female students in the experimental group. Dance aerobics with hip-hop elements was used during the classes in the control group.

Hip-hop as a complex coordination dance sports type presents:

1. High-amplitude intensive movements with a quick change of body parts positions and local movements;

2. Dance styles' synthesis (basic hip-hop school (old, middle, new), locking, popping, dancehall, house, break dance, vogue dance, waacking), which have different tempo rhythmic and movement structure;

3. Usage of specific music when doing the dance routines and combinations;

4. Different rhythmic patterns in the choreographic basis of the composition;

5. Multilevel position change.

Coordination skills improvement method was used in the scope of education process of female students' during the classes within the course "Physical educations and sports". Each class took place twice a week and lasted for 80 minutes. The length and frequency of the classes are determined by the curriculum for "Physical education and sports" of MIREA - Russian Technological University. First year female students18-20 years old, referred to the main health group.

Based on the main principles of the physical education class structure, practical part of our method consisted of a preparatory, main and concluding parts.

\section{Preparatory part:}

- hip-hop dance warm-up activity;

- isolation exercises; 
- exercises aimed to teach bounce \& groove skills.

\section{Main part:}

- Teaching the basic steps and elements of Hip-Hop, Locking, House, Dancehall, Popping technique

- Imroving the basic steps and elements of Hip-Hop, Locking, House, Dancehall, Popping technique

- overall physical training.

Concluding part:

- flexibility development exercises;

- breathing exercises.

The main components of a hip-hop dance class are:

1. Standard repetition teaching method with a variable component

2. Education material differentiation based on the class goals

3. Steps combinations, where movement transition and connecting elements and movements are used

4. Improvisational direction

5. Series of music rtythmical education

6. Individual work.

To estimate the efficiency of the offered method of coordination skills improvement based on hip-hop, we repeated the testing, which included evaluation of lateral dominance level manifestation and arms and legs coordination (Table 1,2).

Table 1. Results of the control and experimental groups based on «Juggling» test before and after the experiment conduction.

\begin{tabular}{|c|c|c|c|c|c|c|}
\hline \multirow{2}{*}{} & \multicolumn{6}{|c|}{ Juggling (number of times) } \\
\cline { 2 - 8 } & \multicolumn{2}{|c|}{$\begin{array}{c}\text { «Right hand» } \\
(\mathbf{x} \pm \sigma)\end{array}$} & \multicolumn{2}{c|}{$\begin{array}{c}\text { «Lef hand» } \\
(\mathbf{x} \pm \sigma)\end{array}$} & \multicolumn{2}{c|}{$\begin{array}{c}\text { Difference } \\
\text { (number module) }\end{array}$} \\
\cline { 2 - 8 } & Before & After & Before & After & Before & After \\
\hline $\begin{array}{c}\text { CONTROL } \\
\text { GROUP }\end{array}$ & $33 \pm 0.5$ & $\begin{array}{c}32.8 \pm 0.5 \\
(\mathrm{p}>0,05)\end{array}$ & $29.3 \pm 0.5$ & $\begin{array}{c}31.7 \pm 0.6 \\
(\mathrm{p}<0,05)\end{array}$ & $3.8 \pm 0.6$ & $\begin{array}{c}2.1 \pm 0.5 \\
(\mathrm{p}<0,05)\end{array}$ \\
\hline $\begin{array}{c}\text { EXPERIMENTAL } \\
\text { GROUP }\end{array}$ & $32.1 \pm 0.6$ & $\begin{array}{c}37.7 \pm 0.8 \\
(\mathrm{p}<0,05)\end{array}$ & $28.4 \pm 0.5$ & $\begin{array}{c}36.6 \pm 0.8 \\
(\mathrm{p}<0,05)\end{array}$ & $4.1 \pm 0.4$ & $\begin{array}{c}1.1 \pm 0.6 \\
(\mathrm{p}<0,05)\end{array}$ \\
\hline
\end{tabular}

Table 2. Results of the control and experimental groups based on «Coordination routine» test before and after the experiment conduction.

\begin{tabular}{|c|c|c|c|c|c|}
\hline & & «legs» & «arms» & «legs + arms» & $\begin{array}{c}\text { Total } \\
\text { points }\end{array}$ \\
\hline CONTROL & Before & $2.6 \pm 0.1$ & $2.57 \pm 0.1$ & $2.1 \pm 0.2$ & $7.3 \pm 0.2$ \\
\cline { 2 - 6 } GROUP & After & $\begin{array}{c}4.5 \pm 0.2 \\
(\mathrm{p}<0,05)\end{array}$ & $\begin{array}{c}4.5 \pm 0.1 \\
(\mathrm{p}<0,05)\end{array}$ & $\begin{array}{c}4.3 \pm 0.1 \\
(\mathrm{p}<0,05)\end{array}$ & $\begin{array}{c}13.3 \pm 0.3 \\
(\mathrm{p}<0,05)\end{array}$ \\
\hline \multirow{2}{*}{$\begin{array}{c}\text { EXPERIMENTAL } \\
\text { GROUP }\end{array}$} & Before & $2.6 \pm 0.1$ & $2.6 \pm 0.1$ & $2.3 \pm 0.2$ & $7.4 \pm 0.3$ \\
\cline { 2 - 6 } & After & $3.6 \pm 0.2$ & $\begin{array}{c}2.9 \pm 0.1 \\
(\mathrm{p}>0,05)\end{array}$ & $\begin{array}{c}2.6 \pm 0.1 \\
(\mathrm{p}<0,05)\end{array}$ & $\begin{array}{c}9.1 \pm 0.3 \\
(\mathrm{p}<0,05)\end{array}$ \\
\hline
\end{tabular}

Based on the indicator, defining the level of hands coordination and lateral dominance manifestation («Juggling» testing task) (Table 1), experimental group showed reliable positive changes $(\mathrm{p}<0,05)$ for all 3 main parameters: in juggling with the right hand the 
average number of conducted throws changed by $17.4 \%$, in juggling with the left hand, there was about $28.9 \%$ growth, and the difference between juggling with the right and left hands decreased by $74.1 \%$. As for results, obtained in the control group, unreliable changes $(p>0,05)$ were revealed in juggling with the right hand - the change amounts to $0.7 \%$, reliable change $(p<0,05)$ in left hand juggling $8.2 \%$, and also reliable change $(p<0,05)$ in decrease of the difference between the right and left hand juggling $-45 \%$.

In the testing task «Coordination routine» the following changes were revealed: statistically significant change $(\mathrm{p}<0,05)$ of the «leg» indicator in the control group after usage of dance aerobics with hip-hop elements in the process of physical education of female students, was 1.1 points, whereas in the experimental group this indicator increased by 1.9 points. The change of the «hand» indicator in the control group wasn't statistically reliable $(\mathrm{p}>0,05)$ and comprised 0.3 points, however, in experimental group, changes are statistically reliable $(p<0,05)$ and comprise 1.9 points. Relatively small reliable growth $(p<0,05)$ was revealed in the control group for indicator «legs + arms» -0.3 points, whereas in the experimental group under the obtained statistically reliable data $(p<0,05)$ changes comprised 2.2 points. Based on overall points indicator in the control group there emerged reliable changes $(p<0,05)$ by 1.7 points, in the experimental group the changes are also reliable $(\mathrm{p}<0,05)$, however, the growth was about 6 points.

\section{Conclusions}

1. As the result of evaluation of the initial manifestation of lateral dominance among the female students of non-sports university, functional motor asymmetry of predominant rightside type was revealed, which is not a physiological standard of brain hemispheres activity.

2. To increase education process efficiency in the area of physical education in the university, we elaborated and used in practice a method based on the hip-hop tools during the education process, the method proved to be efficient and allows to decrease the level of lateral dominance manifestation, to improve legs and arms coordination, which, in its turn, enables a more optimal functioning and activity of the central nervous system.

\section{References}

1. Abaskalova, N.P. and Pyzh'yanova, N.N. (2002) Functional interhemispheric asymmetry, training and health, Vestnik BGPU, No 1. 23 - 34.

2. Aleksandrova, V. A. (2012) The use of classical (basic) aerobics to assess the coordination abilities of dancers of sports ballroom dancing, Teoriya i praktika fizicheskoj kul'tury, No1. 11.

3. Serikova, Yu. N. and Aleksandrova, V. A. (2017) Analysis of the level of coordination abilities of female students of an unsportsmanlike university 18-20 years old, materials of the scientific conference: interregional final scientific conferences of students "Student Science" and "Young Scientists of SCIPC" March 15-17, 2017, April 12-14, 2017, Moscow. 240-244.

4. Bragina, N.N and Dobrohotova, T.A. The functional asymmetry of the human, 2nd ed., revised and ext. Moscow: Medicine. 240.

5. Mazikin, I.M., Lapkin, M.M., Voshinina, N.A., and Proshlyakov, V.D. (2016) Influence of the brain lateral organization profile on efferectivencess of sporting activity of a human and methods of its identification, Ryazan. 117-126.

6. Efimova, I. V. (1996) Hemispheric asymmetry of the brain and motor skills, Human physiology, No 22 (1). 35- 39. 
7. Maksimenko, A.M. (2001) Bases of the theory and methods of physical culture. textbook for university students, 4-y filial Voenizdata. 319.

8. Matveev, L.P. (2008) The theory and methods of physical culture, Moscow: Fizkul'tura i sport, Sport Akadem Press, No3. 544.

9. Matova, M.A. (1980) Functional asymmetry and symmetry of spatial perception in athletes of different specialties, Theory and Practice of Physical Culture, No11. 2-5.

10. Platonov, V.N. (2002). Coordination of the athlete and methods for improving it. Kiev: GIFK. 52.

11. Sologub, E.B and Tajmazov, V.A. (2000) Sports Genetics: tutorial, Moscow: TerraSport. 127.

12. Homskaja, E.D.( 2005) Neuropsychology, SPb, Piter. 496.

13. Fudin, N.A, Klassina, S.Ja, and Pigareva, S.N. (2014) Features of the system organization of physiological functions at various stages of increasing physical activity by persons engaged in physical culture and sports, Sports Medicine: Science and Practice, No3. 14-18.

14. Annet, M. (1995), The rightshift theory of a genetic balanced polymorphism for cerebral dominance and cognitive processing, Current Phychol. Cognit., Vol. 14 No. 5. 427-480.

15. Bishop, D. (1990), Handedness and developmental disorders, Oxford. 